\title{
Análisis del Comportamiento Mecánico del Tejido Subyacente a las Tuberosidades Isquiáticas por Medio del Método del Elemento Finito
}

\author{
Analysis of Mechanical Behavior of the Underlying Tissue \\ to the Ischial Tuberosities Using Finite Element Method
}

\begin{abstract}
Víctor M. Araujo-Monsalvo*; José J. Silva-Lomelí*; ; Aylenid Alemán-Pérez**; Víctor M. Domínguez-Hernández*; Marcos Martínez-Cruz ${ }^{* * *}$; Elisa Martínez-Coria***** \& Diana A. Gayol-Mérida******
\end{abstract}

ARAUJO-MONSAlVO, V. M.; SILVA-LOMELÍ, J. J.; ALEMÁN-PÉREZ, A.; DOMÍNGUEZ-HERNÁNDEZ, V. M.; MARTÍNEZ-CRUZ, M.; MARTÍNEZ-CORIA, E. \& GAYOL-MÉRIDA, D. A. Análisis del comportamiento mecánico del tejido subyacente a las tuberosidades isquiáticas por medio del método del elemento finito. Int. J. Morphol., 34(3):1142-1147, 2016.

RESUMEN: Las úlceras por presión son las complicaciones secundarias más comunes a una lesión medular, las cuales ponen en riesgo tanto la salud como la vida de quienes las padecen. Las úlceras por presión más comunes en lesionados medulares aparecen en la región pélvica, principalmente en las tuberosidades isquiáticas (TI's). Una estrategia usada en la clínica es medir la presión generada entre el paciente y la superficie donde se encuentra para evaluar el riesgo que representa dicha superficie para el desarrollo de úlceras por presión sin embargo, este tipo de mediciones superficiales no garantizan que la presión en los tejidos internos subyacentes a prominencias óseas sea inocua. Con el fin de estudiar los mecanismos de formación de úlceras por presión, se realizó el análisis de un modelo de pelvis y tejido subyacente por medio del Método de los Elementos Finitos (MEF). De esta manera se puede estudiar el comportamiento de las TI's sobre su tejido circundante, así como analizar los efectos biomecánicos que provocan las úlceras. Se construyó el modelo computacional por medio de un software de CAD (Computing Aided Design) de la pelvis a partir de cortes tomográficos. El modelo fue exportado al software COMSOL y se analizaron seis casos de estudio: un análisis de la pelvis sobre bloques de tejido sano y cinco casos más, los cuales simulan lesiones en el tejido con distintas profundidades, representando úlceras superficiales e internas. Los resultados mostraron que los puntos de máximo esfuerzo, en todas las pruebas, se localizan justo debajo de la TIs además se encontró que las lesiones internas presentan mayores esfuerzos y deformaciones, los cuales pueden ser precursores de daño en el tejido.

PALABRAS CLAVE: Úlcera por presión; Lesión de tejido profundo; Análisis por elementos finitos.

\section{INTRODUCCIÓN}

Según la NPUAP (The National Pressure Ulcer Advisory Panel, 2007), las úlceras por presión son lesiones en el tejido causadas por presión constante ejercida por prominencias óseas durante un periodo prolongado, lo cual causa obstrucción capilar y daña el tejido subyacente.

Las úlceras por presión son un problema de salud que afecta aproximadamente al 0,5\% de la población total, según Donini et al. (2005). La causa primaria de las úlceras por presión es la presión externa por tiempo prolongado sobre las prominencias óseas, sin embargo, la patogénesis de las úlceras por presión es multifactorial. Esta complicación afecta a personas que, debido a enfermedad o a su condición física, permanecen sentadas o acostadas la mayor parte del tiempo, tales como las personas de edad avanzada y los lesionados medulares.

Según Donini, la prevalencia de éstas va de 1 a $18 \%$ en pacientes hospitalizados y se ha estimado que $50 \%$ de las personas que sufren cuadriplejia y más del $30 \%$ de la po-

\footnotetext{
* Laboratorio de Biomecánica, Instituto Nacional de Rehabilitación, Ciudad de México, México.

** Unidad Profesional Interdisciplinaria y Tecnologías Avanzadas, Instituto Politécnico Naciona, Ciudad de México, México.

*** Facultad de Ingeniería, Universidad Nacional Autónoma de México, Ciudad de México, México.

***** Servicio de Tomografía Computada, Instituto Nacional de Rehabilitación, Ciudad de México, México.

****** Laboratorio de Ingeniería de Rehabilitación, Instituto Nacional de Rehabilitación, Ciudad de México, México.
} 
blación con paraplejia, requiere de hospitalización debido a problemas relacionados con úlceras por presión. Se estima que entre el $31 \%$ al $79 \%$ de los lesionados medulares desarrolla úlceras por presión (Henzel et al., 2011), siendo estas una de las principales causas de readmisión hospitalaria, pudiendo requerir en promedio 180 días de cuidados de enfermería (Bogie \& Bader, 2005).

De acuerdo con Bogie et al. (1995) el $47 \%$ de las úlceras por presión que presentan los lesionados medulares se encuentran en la zona de las tuberosidades isquiáticas y sacro, esto derivado de que puedan pasar hasta 16 horas sentados en una silla de ruedas (Sprigle et al., 1990). Se han sugerido diversos métodos para la prevención de úlceras por presión en pacientes susceptibles a padecerlas. Entre las estrategias preventivas de formación de úlceras se incluyen cambio de posición cada determinado tiempo para compensación de presiones, educación preventiva, sillas de ruedas personalizadas y uso de superficies de soporte (Regan et al., 2009). Según Sprigle et al., uno de los medios más utilizados clínicamente para evaluar la habilidad de una superficie de soporte para la distribución de la carga es la medición de las presiones entre el paciente y el asiento. Sin embargo, mientras las presiones superficiales pueden ser medidas, los esfuerzos en los tejidos internos cercanos a las prominencias óseas, son desconocidos.

El uso de modelos computacionales para simular el comportamiento de las cargas internas al tejido, pueden ser aplicados para el estudio de un tipo de úlcera por presión reconocida en años recientes por la NPAUP/EPAUP, como sospecha de daño tisular profundo (DTI por sus siglas en inglés Deep Tissue Injury) (The National Pressure Ulcer Advisory Panel). Xiao et al. (2014) resalta que este tipo de lesiones representan un reto clínico debido a que el daño tisular subcutáneo en principio puede no ser notado en la superficie del tejido. En un estudio realizado por VanGilder et al. (2010), en Estados Unidos encontraron una prevalencia mayor de casos de DTI's, de otro grado de úlceras en estadios avanzados (VanGilder et al.), también encontraron que en lesionados medulares, entre las zonas donde existe un mayor riesgo de desarrollar DTI's son la zona sacra (19 $\%$ ) y las tuberosidades isquiáticas (13\%), lo que hace prioritario el estudio del desarrollo de este tipo de úlceras, con el fin de apoyar a su prevención y tratamiento.

Este trabajo, se enfoca al estudio del comportamiento de desplazamientos y esfuerzos que interactúan entre las tuberosidades isquiáticas y sus tejidos blandos subyacentes, con el fin de entender el fenómeno que genera la formación de las úlceras por presión, empleando el método de los elementos finitos (MEF) en modelos 3D. Se simularon seis casos: 1) tejido sano, 2) lesión en el tejido interno subyacente a la tuberosidad isquiática, 3) lesión que afecta en su totalidad el tejido blando sin afectar la capa de la piel, 4) lesión que afecta en su totalidad al tejido incluyendo la capa de la piel, 5) lesión externa que sólo afecta a la capa de piel, y 6) lesión externa que afecta la capa de la piel y parte del tejido blando.

\section{MATERIAL Y MÉTODO}

Procesamiento de cortes tomográficos: Para construir el modelo 3D de la pelvis se utilizaron los archivos obtenidos del sistema de información médica y telemedicina PACSINR (Picture Archiving and Communication System) del Instituto Nacional de Rehabilitación Luis Guillermo Ibarra Ibarra (Cd. de México, México). Estas imágenes corresponden a un sujeto de sexo masculino de $70 \mathrm{~kg}$ de peso.

Se obtuvo un total de 364 cortes tomográficos con una distancia entre ellos de $0,635 \mathrm{~mm}$, desde la cresta ilíaca hasta la parte distal del isquion. Las imágenes fueron exportadas en formato DICOM (Digital Imaging and Communication in Medicine) para su posterior segmentación (Fig. 1).

Se utilizó el software SolidWorks (Dassault Sistems, Vélizy-Villacoublay, Francia) para procesar las imágenes tomográficas y de este modo se obtuvieron 30 puntos por cada corte (Fig. 2), los cuales describen sólo la geometría externa de la pelvis. A estos puntos se los denomina keypoints (coordenadas).

Construcción de modelo 3D de elementos finitos: El set de keypoints fue importado al software COMSOL

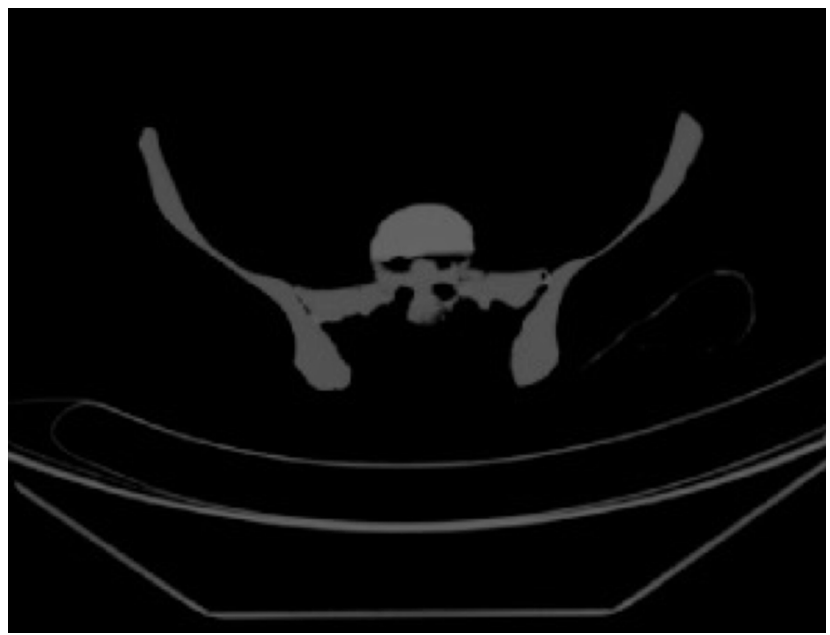

Fig. 1. Imagen tomográfica. Corte transversal de la parte superior de la pelvis. 
ARAUJO-MONSALVO, V. M.; SILVA-LOMELÍ, J. J.; ALEMÁN-PÉREZ, A.; DOMÍNGUEZ-HERNÁNDEZ, V. M.; MARTÍNEZ-CRUZ, M.; MARTÍNEZ-CORIA, E. \& GAYOLMÉRIDA, D. A. Análisis del comportamiento mecánico del tejido subyacente a las tuberosidades isquiáticas por medio del método del elemento finito. Int. J. Morphol., 34(3):1142-1147, 2016.

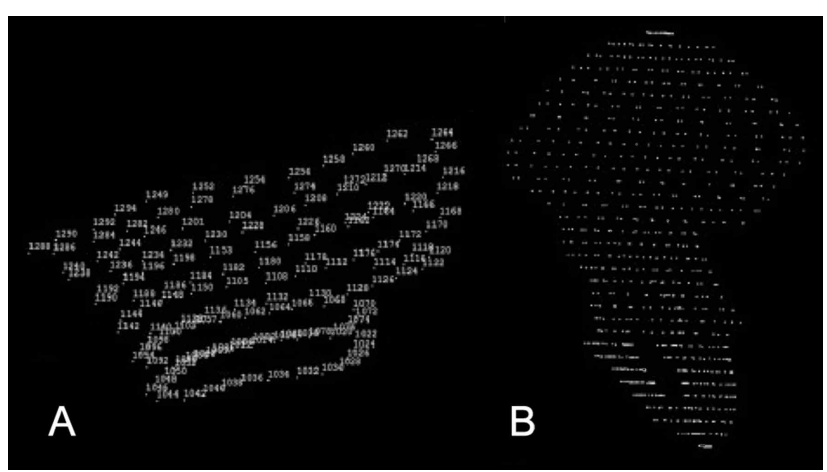

Fig. 2. Imagenes de la parte derecha del modelo. A) Puntos de control numerados y B) Puntos de control (Keypoints).

Multiphysics Inc., Burlington, MA, USA. Versión 5.2, para obtener el modelo 3D. Con los keypoints se formaron líneas, posteriormente se generaron áreas, las áreas fueron unidas manualmente para generar volúmenes, obteniendo al final un sólido de la pelvis (Figs. 3, 4). Para el presente análisis, las propiedades de los tejidos fueron tomados como las correspondientes a materiales elásticos, isotrópicos y lineales, con el fin de simplificar los casos de estudio (Lin et al., 2004; Majumder et al., 2005; Li et al., 2013; Xiao et al.) (Tabla I). Así también, se realizó una prueba de convergencia del modelo, para evaluar el efecto del tamaño de malla y garantizar que este aspecto no afecte los resultados. El mode- lo se construyó utilizando elementos Solid92 (tetraedros de 10 nodos), obteniendo 276,449 elementos con 461,655 nodos.

Casos de estudio. Las condiciones de carga y de frontera fueron las mismas para los seis casos, la carga de $450 \mathrm{~N}$ que corresponde al peso del torso de un hombre de $70 \mathrm{~kg}$ (Matsuo et al., 2011), esta carga fue aplicada en la base del sacro, y se establecieron elementos de contacto entre el bloque de tejido blando y las tuberosidades isquiáticas. La restricción de movimiento se aplicó en la parte inferior de los bloques que simulan el tejido blando.

Para analizar el comportamiento mecánico de la pelvis sobre el tejido blando, debajo de las tuberosidades isquiáticas se colocaron dos bloques de $60 \times 80 \times 32 \mathrm{~mm}$ que simularon el tejido blando. El bloque izquierdo se definió como el tejido con daño, mientras que el derecho permaneció como el tejido sano, dentro de los bloques se colocaron cilindros, que simularon tanto el tejido sano, como el tejido dañado para los siguientes casos. Para la lesión interna: el cilindró fue de $20 \mathrm{~mm}$ de radio y $17 \mathrm{~mm}$ de alto y se colocó debajo de la punta de la tuberosidad isquiática (Donini et al.). Para la úlcera superficial se agregó al cilindro anterior un cilindro de $20 \mathrm{~mm}$ de radio y $17 \mathrm{~mm}$ de alto en la parte inferior del bloque de tejido, y se sumó un tercer cilindró que correspondió a la capa de piel sus dimensiones fueron $20 \mathrm{~mm}$ de radio y $2 \mathrm{~mm}$ de alto (Fig. 5).
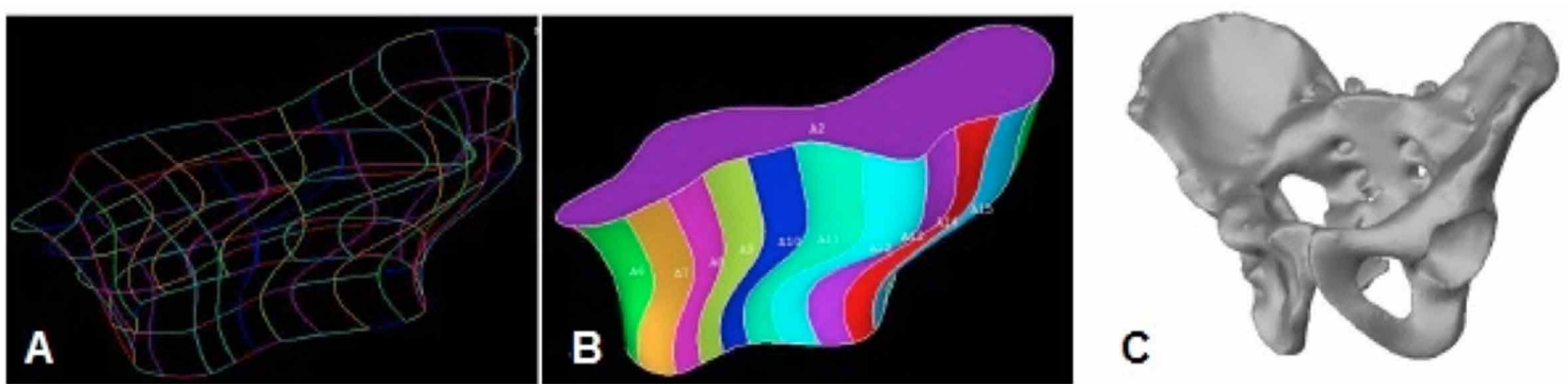

Fig. 3. Formación del modelo 3D. A) Líneas para formar áreas, B) Áreas para formar volúmenes y C) Modelo de la pelvis de estudio.

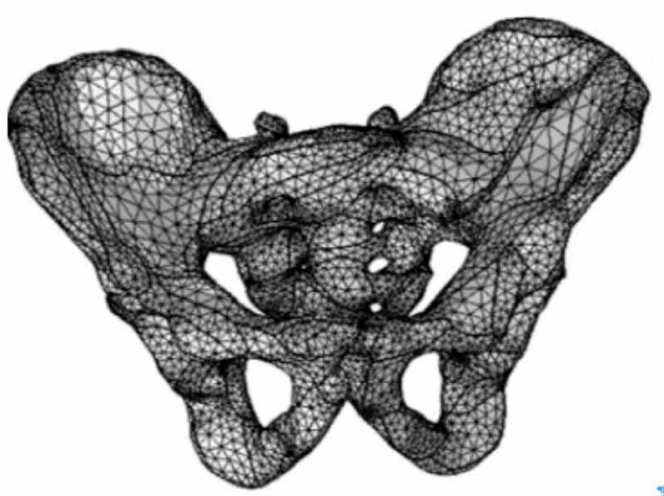

Fig. 4. Mallado del modelo 3D (tetraedros de 10 nodos).

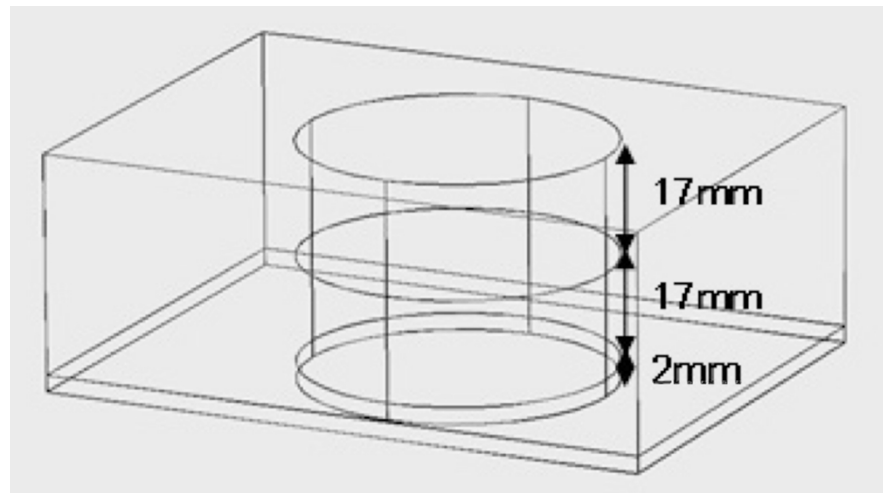

Fig. 5. Aplicación de la carga y condiciones de frontera del modelo 3D. 
ARAUJO-MONSALVO, V. M.; SILVA-LOMELÍ, J. J.; ALEMÁN-PÉREZ, A.; DOMÍNGUEZ-HERNÁNDEZ, V. M.; MARTÍNEZ-CRUZ, M.; MARTÍNEZ-CORIA, E. \& GAYOLMÉRIDA, D. A. Análisis del comportamiento mecánico del tejido subyacente a las tuberosidades isquiáticas por medio del método del elemento finito. Int. J. Morphol., 34(3):1142-1147, 2016.

Tabla I. Propiedades mecánicas de los materiales usados en el modelo 3D.

\begin{tabular}{lccc}
\hline \multicolumn{1}{c}{ Material } & Módulo de Elasticidad E (MPa) & Relación de Poisson & Densidad $\left(\mathbf{k g} / \mathbf{m}^{\mathbf{3}}\right)$ \\
\hline Hueso & $1,70 \mathrm{E}+04$ & 0,3 & 2000 \\
Tejido blando & 20 & 0,49 & 750 \\
Piel & 0,045 & 0,485 & \\
\hline
\end{tabular}

\section{RESULTADOS}

Se obtuvieron los desplazamientos máximos de cada caso de estudio y se graficaron comparando la tuberosidad izquierda (dañada) contra la tuberosidad derecha (sana) (Fig. 6).

Se puede observar que hay mayor desplazamiento en las simulaciones de lesión interna (casos 1 y 2), así como en la que todo el tejido está dañado (caso 3) y que éste se incrementa, conforme aumenta el daño en el tejido.

En las lesiones externas (casos 4 y 5), los desplazamientos son menores, sin embargo, sobrepasan a los calculados en el tejido sano. En este caso también se puede observar que el desplazamiento máximo aumenta entre más daño exista.

Se obtuvo el esfuerzo de von Mises máximo de cada caso de estudio y los resultados se graficaron comparando la tuberosidad izquierda (dañada) y la tuberosidad derecha (sana) (Fig. 7).

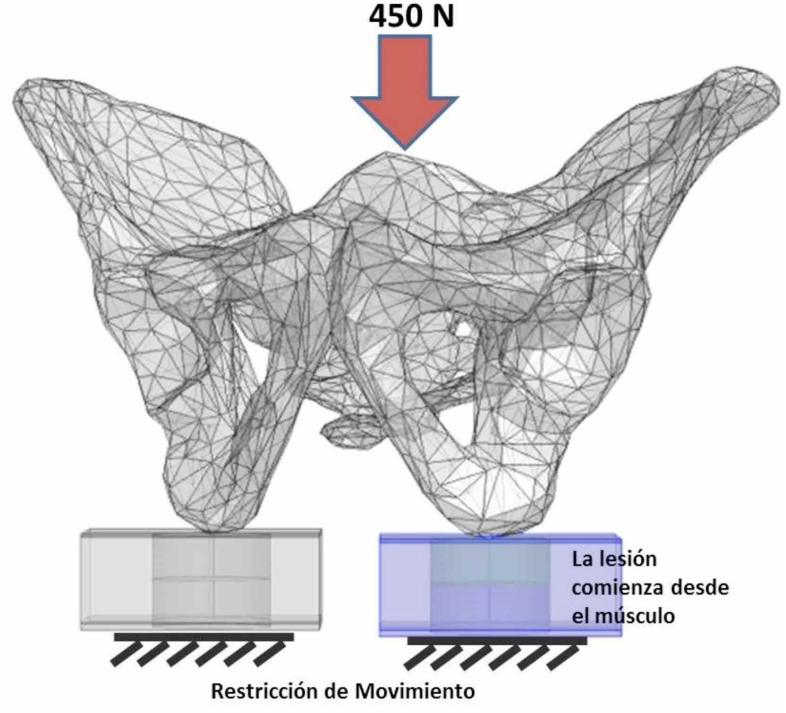

Fig. 6. Adición de bloques de 60x80x32mm que simulan el tejido blando.

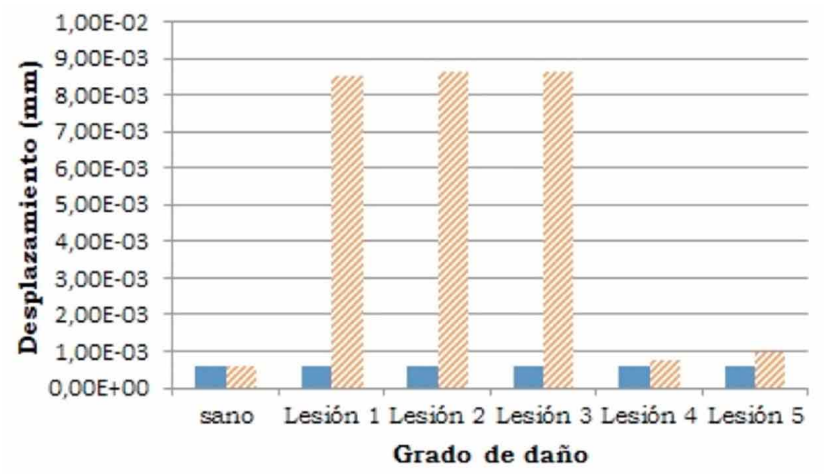

Fig. 7. Desplazamientos de los seis casos de estudio.

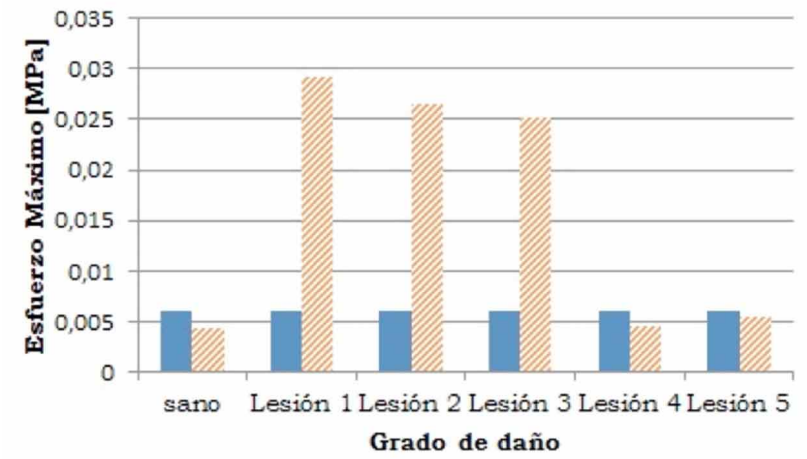

Fig. 8. Esfuerzos máximos de von Mises de los seis casos de estudio.

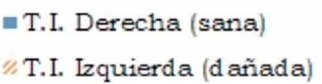

\footnotetext{
- T.I. Derecha (sana)

") T.I. Izquierda (dañada)
} 
ARAUJO-MONSALVO, V. M.; SILVA-LOMELÍ, J. J.; ALEMÁN-PÉREZ, A.; DOMÍNGUEZ-HERNÁNDEZ, V. M.; MARTÍNEZ-CRUZ, M.; MARTÍNEZ-CORIA, E. \& GAYOLMÉRIDA, D. A. Análisis del comportamiento mecánico del tejido subyacente a las tuberosidades isquiáticas por medio del método del elemento finito. Int. J. Morphol., 34(3):1142-1147, 2016.

En el caso de los esfuerzos, se puede notar una diferencia entre la tuberosidad derecha e izquierda incluso cuando ambos tejidos están sanos. Igual que en el caso anterior se puede observar que hay mayor esfuerzo en las simulaciones de lesión interna (casos 1 y 2), así como en la que todo el tejido está dañado (caso 3), sin embargo el valor disminuye conforme aumenta el daño en el tejido (Fig. 8).

En las lesiones externas (casos 4 y 5), los esfuerzos son menores, y no sobrepasan a los calculados en el tejido sano en comparación con la tuberosidad cuyo tejido subyacente no presenta daño, sin embargo, sí sobrepasa al valor calculado cuando la tuberosidad izquierda está sana. En este caso se puede observar que el esfuerzo aumenta entre más tejido dañado exista.

\section{DISCUSIÓN}

El Método del Elemento Finito (MEF) es una herramienta muy útil para analizar escenarios hipotéticos, y que no pueden reproducirse de otra forma. En el presente trabajo se reproduce la geometría de la pelvis.

El tener acceso a un modelo de este tipo puede ser muy ventajoso para los investigadores, así como para los médicos, ya que en un modelo de la pelvis se puede obtener información sobre estudios y análisis en los cuales se requiera conocer el comportamiento biomecánico del tejido subyacente en las primeras etapas del estudio.

En relación con los esfuerzos y desplazamientos obtenidos por el análisis de MEF, se estiman los efectos biomecánicos sobre el tejido subyacente a las tuberosidades isquiáticas, tanto cuando éste se encuentra sano, como cuando aparecen lesiones, ya sean internas o externas, cumpliendo con el objetivo general de este proyecto. Tanto esfuerzos como desplazamientos son mayores en las lesiones internas a comparación de las externas. Esto indica que las lesiones que comienzan desde dentro generan mayores esfuerzos en el tejido desde etapas tempranas, por lo cual, podría dañar más rápido el tejido circundante.

En otros estudios realizados con modelos físicos se ha encontrado que los puntos de esfuerzo se localizan justo debajo de la tuberosidad isquiática. Lo cual concuerda con los trabajos de Gefen \& Levine (2007). Los resultados, reflejan un esfuerzo máximo entre 4.3 y $6 \mathrm{kPa}$ lo cual concuerda con el trabajo de Reddy et al. (1982), quien realizó un modelo físico y midió los esfuerzos aplicando carga, calculando los valores de los esfuerzos máximos entre 5.7 y 10 $\mathrm{kPa}$. Estos resultados demuestran la importancia de estudiar los esfuerzos internos de los sujetos que permanecen en sedestación, ya que se pueden generar esfuerzos internos dañinos, a pesar que las mediciones superficiales no lo reflejen.

Se observa de igual manera que existen diferencias entre los resultados de la tuberosidad derecha y la tuberosidad izquierda aun cuando ambos lados están considerados como sanos. Esto se puede deber a que el modelo está basado en la pelvis de un paciente, dado que no es simétrico, los esfuerzos no toman el mismo valor en ambos lados. También se observó que el desplazamiento aumenta conforme aumenta el daño en el tejido lo cual era lo esperado, ya que, al presentar menor tejido que soporte esa zona, hay un mayor desplazamiento. El valor del esfuerzo máximo disminuye en las lesiones internas, a diferencia de lo que ocurre en las externas. Dado que el esfuerzo va desde 4,54 hasta 6,26 veces, y es mayor cuando la lesión es interna, a comparación de las lesiones externas, éste daña más área, y por ende la carga se reparte en un área mayor. Esto quiere decir que aunque los esfuerzos disminuyan, se daña más tejido.

Los resultados obtenidos concuerdan con los hallazgos clínicos y permiten avanzar en la comprensión del fenómeno complejo que representan las úlceras por presión.

\section{AGRADECIMIENTOS}

Los autores agradecen el trabajo realizado por el técnico Martín Luna Méndez del Servicio de Tomografía Computada del Instituto Nacional de Rehabilitación Luis Guillermo Ibarra Ibarra, al igual agradecemos las facilidades otorgadas por la Unidad Profesional Interdisciplinaria en Ingeniería y Tecnología Avanzadas (Instituto Politécnico Nacional) y a la Escuela Superior de Medicina (Instituto Politécnico Nacional).

ARAUJO-MONSALVO, V. M; SILVA-LOMELÍ, J. J.; ALEMÁN-PÉREZ, A.; DOMÍNGUEZ-HERNÁNDEZ, V. M.; MARTÍNEZ-CRUZ, M.; MARTÍNEZ-CORIA, E. \& GAYOLMÉRIDA, D.A. Analysis of mechanical behavior of the underlying tissue to the ischial tuberosities using finite element method. Int. J. Morphol., 34(3):1142-1147, 2016.

SUMMARY: Pressure ulcers are the most common secondary complication to a spinal cord injury, which endanger both health and life of the patients who suffer them. The most common pressure ulcers in spinal cord injuries occur in the pelvic region, mainly in the ischial tuberosities (ITs). A strategy used in clinic is to quantify the pressure generated between the patient and 
ARAUJO-MONSALVO, V. M.; SILVA-LOMELÍ, J. J.; ALEMÁN-PÉREZ, A.; DOMÍNGUEZ-HERNÁNDEZ, V. M.; MARTÍNEZ-CRUZ, M.; MARTÍNEZ-CORIA, E. \& GAYOLMÉRIDA, D. A. Análisis del comportamiento mecánico del tejido subyacente a las tuberosidades isquiáticas por medio del método del elemento finito. Int. J. Morphol., 34(3):1142-1147, 2016.

the surface, in order to assess the risk posed by that surface for developing pressure ulcers. Despite this, this type of surface measurements does not guarantee that pressure in the internal tissues underlying to bony prominences, to be safe. In order to study the mechanisms of formation of pressure ulcers, an analysis of a model of the pelvis and its underlying tissue was performed using the Finite Element Method (FEM). By this means we can study the behavior of ITs on its surrounding tissue, and at the same time, we analyze the biomechanical effects those cause ulcers. The computational model of the pelvis was built from tomographic slices using CAD software (Computing Aided Design). The model was exported to the finite element software COMSOL and six study cases were analyzed: an analysis of the pelvis on healthy tissue blocks and five more cases, which simulate tissue injury with different depths, representing surface and internal ulcers. The results showed that the maximum stress points in all tests are located just below the ITs it was also found that internal injuries present higher stresses and strains, which can be precursors of tissue damage.

KEY WORDS: Pressure ulcer; Deep Tissue Injury; Finite Element Analysis.

\section{REFERENCIAS BIBLIOGRÁFICAS}

Bogie, K. M.; Nuseibeh, I. \& Bader, D. L. Early progressive changes in tissue viability in the seated spinal cord injured subject. Paraplegia, 33(3):141-7, 1995.

Bogie, K. M. \& Bader, D. L. Susceptibility of Spinal Cord-Injured Individuals to Pressure Ulcers. In: Bader, D. L.; Bouten, C.; Colin, D. \& Oomens, C. W. J. (Eds.). Pressure Ulcer Research: Current and Future Perspective. Berlin, Springer, 2005. pp.7388.

Donini, L. M.; De Felice, M. R.; Tagliaccica A.; De Bernardini, L. \& Cannella, C. Comorbidity, frailty, and evolution of pressure ulcers in geriatrics. Med. Sci. Monit., 11(7):CR326-36, 2005.

Gefen, A. \& Levine, J. The false premise in measuring body-support interface pressures for preventing serious pressure ulcers. $J$. Med. Eng. Technol., 31(5):375-80, 2007.

Henzel, M. K.; Bogie, K. M.; Guihan, M. \& Ho, C. H. Pressure ulcer management and research priorities for patients with spinal cord injury: consensus opinion from SCI QUERI Expert Panel on Pressure Ulcer Research Implementation. J. Rehabil. Res. Dev., 4(3):xi-xxxii, 2011.

Li, S.; Zhang, Z. \& Wang, J. A New Custom-Contoured Cushion System Based on Finite Element Modeling Prediction. J. Mech. Med. Biol., 13(4):1350051, 2013.

Lin, F.; Moran, B.; Bankard, J.; Hendrix, R. \& Makhsous, M. FEM model for evaluating buttock tissue response under sitting load. Conf. Proc. I. E. E. E. Eng. Med. Biol. Soc., 7:5088-91, 2014.
Majumder, S.; Roychowdhury, A. \& Pal, S. A Finite Element Study on the Behavior of Human Pelvis Under Impact through Car Door. Berichte der Bundesanstalt Fuer Strassenwesen. Unterreihe Fahrzeugtechnik, 55, 2005. pp.196-203.

Matsuo, J.; Sugama, J.; Sanada, H.; Okuwa, M.; Nakatani, T.; Konya, C. \& Sakamoto, J. Development and validity of a new model for assessing pressure redistribution properties of support surfaces. J. Tissue Viability, 20(2):55-66, 2011.

The National Pressure Ulcer Advisory Panel. NPUAP Pressure Ulcer Stages/Categories. Washington D. C., The National Pressure Ulcer Advisory Panel, 2007. Disponible en: http:// www.npuap.org/wp-content/uploads/2012/01/NPUAPPressure-Ulcer-Stages-Categories.pdf

Reddy, N. P.; Patel, H.; Cochran, G. V. \& Brunski, J. B. Model experiments to study the stress distributions in a seated buttock. J. Biomech., 15(7):493-504, 1982.

Regan, M. A.; Teasell, R. W.; Wolfe, D. L.; Keast, D.; Mortenson, W. B.; Aubut, J. A. \& Spinal Cord Injury Rehabilitation Evidence Research Team. A systematic review of therapeutic interventions for pressure ulcers after spinal cord injury. Arch. Phys. Med. Rehabil., 90(2):213-31, 2009.

Sprigle, S.; Chung, K. C. \& Brubaker, C. E. Reduction of sitting pressures with custom contoured cushions. J. Rehabil. Res. Dev., 27(2):135-40, 1990.

VanGilder, C.; MacFarlane, G. D.; Harrison, P.; Lachenbruch, C. $\&$ Meyer, $\mathrm{S}$. The demographics of suspected deep tissue injury in the United States: an analysis of the International Pressure Ulcer Prevalence Survey 2006-2009. Adv. Skin Wound Care, 23(6):254-61, 2010.

Xiao, D. Z.; Wu, S. Y. \& Mak, A. F. Accumulation of loading damage and unloading reperfusion injury--modeling of the propagation of deep tissue ulcers. J. Biomech., 47(7):165864, 2014.

Dirección de Correspondencia:

Diana Alicia Gayol Mérida

Ingeniería de Rehabilitación

Instituto Nacional de Rehabilitación

Luis Guillermo Ibarra Ibarra

Calz. México-Xochimilco No. 289

Col. Arenal de Guadalupe

Delegación Tlalpan CP. 14389

MÉXICO

Email: dgayol@inr.gob.mx dgayolmerida@gmail.com
Recibido : 19-05-2016

Aceptado: 12-07-2016 